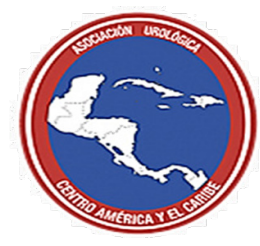

Asociación Urológica de Centroamérica y el Caribe

\section{DISLOCACIÓN TESTICULAR. REVISIÓN DE LA LITERATURA. A PROPÓSITO DE UN CASO}

\section{Testicular dislocation. Review of the literature. Report of one case.}

\section{Autores}

\section{Andrés Salvador Escobar Gómez ${ }^{(1)}$, Carlos Gonzalo Estrada Pazos ${ }^{(2)}$ Luis Alberto Contreras Morales ${ }^{(3)}$ \& Mario José Jo Bonila ${ }^{(4)}$}

(1) Médico Residente V de Cirugía General - Instituto Guatemalteco de Seguridad Social

(2) Jefe de servicio de Urología - Hospital de accidentes "El Ceibal" - Instituto Guatemalteco de Seguridad Social

(3) Médico Residente V de Cirugía General - Instituto Guatemalteco de Seguridad Social

(4) Médico Residente III de Cirugía General - Instituto Guatemalteco de Seguridad Social

Correspondencia:30 calle 7-80 zona 11. Colonia Granai Towson I, Guatemala. Tel. 5756-6111

Correo electrónico: andrescobargo@hotmail.com

DOI: https://doi.org/10.54212/27068048.v9i1.22

Esta investigación fue financiada con recursos propios. Declaramos no tener ningún conflicto de intereses

Fecha de envío: 15/05/2021

Fecha de aceptación: 12/06/2021

Fecha de publicación: 30/06/2021

\section{RESUMEN}

OBJETIVO: Presentar el caso clínico de un paciente con dislocación testicular derecha, la cual ameritó exploración inguinal y orquidopexia transescrotal derecha. ANTECEDENTES: La dislocación testicular es poco frecuente, y se han reportado aproximadamente 200 casos en la literatura médica desde su primera descripción en 1818; en Guatemala solamente se cuenta con una publicación respecto al tema, lo que hace que el caso clínico presentado aporte información respecto al tema. MATERIALES Y MÉTODOS: Se presenta el caso con fotografías clínicas, de un paciente joven, quien sufre accidente en motocicleta, contusionando la región genital contra el tanque de gasolina, provocando la dislocación testicular derecha, siendo manejado de forma quirúrgica. RESULTADOS: En los pacientes que se realiza el diagnóstico temprano, presentan adecuada evolución post quirúrgica, sin complicaciones a largo plazo en su seguimiento. CONCLUSIONES: Pese a que es una patología poco frecuente, el aumento del uso de la motocicleta como medio de transporte en nuestro país, predispone a un incremento en las lesiones de los genitales externos.

\section{ABSTRACT}

OBJECTIVE: To present the clinical case of a patient with right testicular dislocation, which warranted inguinal exploration and right transscrotal orchidopexy. BACKGROUND: Testicular dislocation is rare, and approximately 200 cases have been reported in the medical literature since its first description in 1818; In Guatemala there is only one publication on the subject, which means that the clinical case presented provides information on the subject. MATERIALS AND METHODS: The case is presented with clinical photographs of a young patient, who had a motorcycle accident suffering a direct injury to the genital region against the gas tank, causing the right testicular dislocation, being handled surgically. RESULTS: In patients with early diagnosis, they present adequate post-surgical evolution, without longterm complications during follow-up. CONCLUSIONS: Although it is a rare disease, the increase in the use of motorcycles as a means of transportation in our country predisposes an increase in injuries to the external genitalia. 


\section{PALABRAS CLAVE}

trauma testicular, dislocación testicular, urología, orquidopexia

\section{INTRODUCCIÓN}

La dislocación testicular traumática es una patología poco frecuente, la cual se define como desplazamiento del testículo y sus anexos, previamente situados a nivel intraescrotal, hacia una localización ectópica (1).

Descrito por primera vez por Claubry en 1818(1-3), quien reporta una posición anormal de los testículos en la cavidad abdominal, posterior a una compresión en la parte inferior de la pared abdominal; son pocos los casos que se documentan a nivel mundial de esta patología, principalmente por su diagnóstico tardío y la escasa documentación y reportes de caso. Aportamos al conocimiento general, el siguiente caso clínico que se presenta en el servicio de urología de nuestro hospital de práctica, haciendo una revisión de la literatura más reciente a fin de mejorar su diagnóstico y manejo médico-quirúrgico, y así disminuir las complicaciones que conlleva el retraso del tratamiento.

\section{CASO CLÍNICO}

Se presenta a la unidad de emergencia paciente masculino de 17 años de edad, quien sufre accidente de tránsito en motocicleta, presentando posterior dolor agudo escrotal que no alivia con analgésicos, lo que lo hace consultar 3 horas después del trauma.

A su ingreso, paciente sin lesiones corpóreas traumáticas asociadas, con dolor a nivel testicular derecho, presencia de testículo izquierdo en bolsa escrotal, con adecuado desarrollo para la edad del paciente; y una tumoración a nivel inguinal derecha, no móvil, dolorosa, renitente, lo que hace sospechar que presenta

\section{KEYWORDS}

testicular trauma, testicular dislocation, urology, orchidopexy

dislocación testicular. No se identifican contusiones o laceraciones en el sistema genitourinario, presentando micción espontánea, sin hematuria. Se solicita ultrasonido escrotal, observándose solamente testículo izquierdo en adecuada posición, de características normales, y ausencia de testículo contralateral; se realiza barrido a nivel inguinal derecho, localizando testículo derecho, sin lesión estructural de la túnica albugínea, con parénquima homogéneo, sin áreas de contusión o hematomas y con adecuado flujo vascular a la aplicación de Doppler color tanto del testículo como del cordón espermático derecho. Estudios de gabinete dentro de límites normales, al igual que los marcadores tumorales (AFP, LDH y FA), descartando afección tumoral asociada. Se decide llevar a sala de operaciones de forma urgente, realizando incisión suprainguinal, localizando testículo de aproximadamente $3.3 \mathrm{x}$ $4.5 \mathrm{cms}$ a nivel suprafascial (Fig. 1), por encima del conducto inguinal derecho, con túnica albugínea indemne, sin hematomas, cordón espermático sin hematomas, contusiones o laceraciones (Fig 2.); se realiza orquidopexia transescrotal con sutura multifilamento absorbible, y se realiza cierre de ambas heridas por planos.

Figura 1. Localización de testículo a nivel suprafascial, por encima del canal inguinal. Se localizó el testículo de forma manual y se realizó incisión suprainguinal.

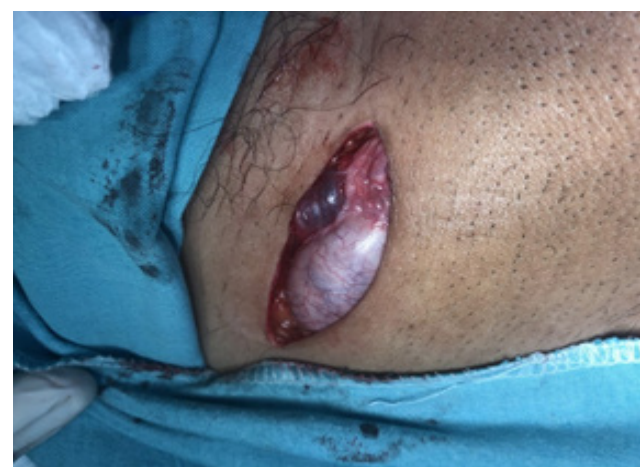


Figura 2. Se exteriorizó en su totalidad el testículo y cordón espermático, el cual, sin lesiones, por lo que se realiza orquidopexia transescrotal.

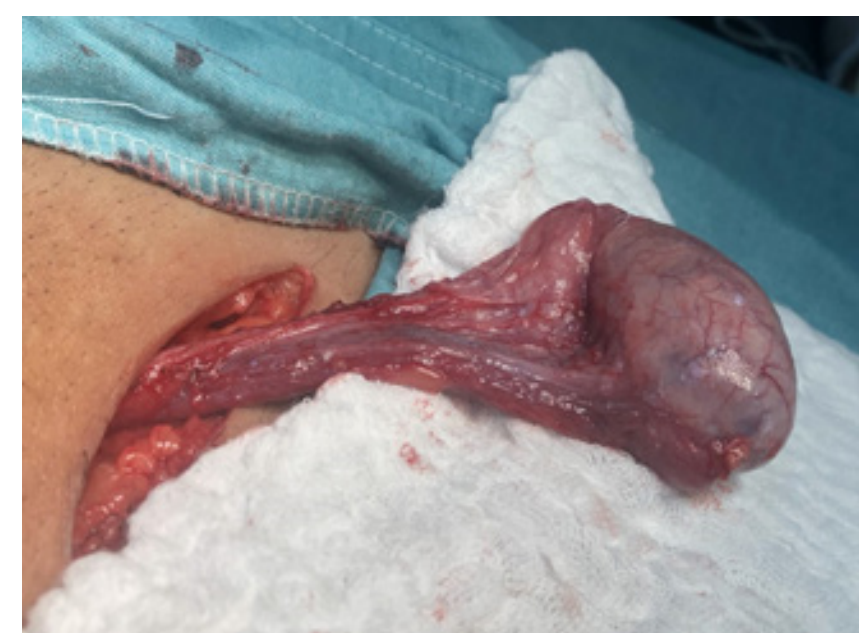

Paciente con adecuada evolución en su post operatorio, tolerando procedimiento, por lo que egresa a las 48 horas de su ingreso. Se realiza seguimiento por consulta externa a las 4 semanas y 4 meses, con ultrasonografía Doppler color y marcadores tumorales, sin presentar complicaciones $u$ alteraciones

\section{DISCUSIÓN}

La dislocación testicular se define como el desplazamiento del testículo, que se encuentra previamente situado a nivel intra-escrotal, hacia una posición ectópica(1) y se presenta como una rara complicación del trauma escrotal contuso(2).

En revisiones recientes, se estima que menos de 200 casos han sido reportados en la literatura mundial, siendo principalmente reportes asiáticos $(2,6)$, españoles $(3,10) y$ estadounidenses(4). En Guatemala, solamente se cuenta con el reporte de un caso del hospital de especialidades de occidente(5), sin embargo, en nuestro hospital se presenta al menos un caso anual, contando aproximadamente con una serie de 22 casos, a quienes se les da seguimiento continuo, sin presentar complicaciones a largo plazo.
La mayoría de los casos son el resultado de lesiones sufridas durante accidentes de motocicleta, cuando el conductor es impulsado hacia adelante, impactando el periné y el escroto contra el tanque de gasolina o el manubrio, presionando el testículo afuera de su localización escrotal normal, $(2,6)$ no obstante los traumas testiculares contusos secundarios a deportes como futbol, béisbol, y ciclismo son otras causas a considerar. Pese a que no se cuenta con datos estadísticos reales sobre la incidencia de esta patología en nuestro país, es de importancia clínica el conocimiento de su manejo médico-quirúrgico, debido al aumento del uso de las motocicletas como medio de transporte, predisponiendo al aumento de accidentes y por ende aumento del riesgo de diversas lesiones traumáticas genitourinarias. A pesar que los genitales externos tienen un riesgo alto de lesiones por su localización extracorpórea, los testículos se encuentran relativamente protegidos por varios mecanismos, como su movilidad característica dentro del escroto, la piel escrotal que provee una elasticidad razonable que permite que las estructuras internas se deslicen y alejen del punto de trauma y la túnica albugínea que funciona como una defensa física fibrosa resistente contra fuerzas de tensión(7). Factores predisponentes a la dislocación testicular han sido reportados, como la existencia previa de hernia inguinal indirecta, un anillo inguinal externo amplio, atrofia testicular y el reflejo cremasté$\operatorname{rico}(6,8,12)$

La dislocación se encuentra en función de la fuerza traumática, de tal forma que, si es menor de $50 \mathrm{~kg}$, se produce una dislocación, mientras que, si es mayor, existirá una ruptura de la túnica albugínea al sobrepasar su resistencia tisular(3). La mayor parte de los casos se presenta de forma unilateral, pero hasta un tercio puede presentarse de forma bilateral(6). El testículo dislocado puede ser desplazado a lo largo de un círculo cuyo radio se encuentra dado por el cordón espermático que se centra a nivel del anillo inguinal externo(9). Las dislocaciones pueden ser superficiales o profundas, siendo la primera la más frecuente, en la cual el testículo se aloja en el tejido subcu- 
táneo, de forma suprafascial, y en el trayecto de su migración, rompe fibras en las fascias espermáticas, creando un ojal que permite el paso en un sentido, pero no en el inverso cuando cesan las fuerzas traumáticas, lo que dificulta su reducción espontánea(1,10).

Schwartz, describió las posibles localizaciones del testículo dislocado, siendo la inguinal superficial la más frecuente $(50 \%)$, seguidas por la púbica $(18 \%)$, peneana $(8 \%)$, canalicular $(8 \%)$, y abdominal (6\%); se mencionan la localización acetabular, perineal crural y femoral como sitios de dislocación poco frecuentes(10).

Los pacientes reportados en la literatura usualmente presentan lesiones mayores asociadas, que incluso pueden poner en riesgo la vida, por lo que muchas veces el diagnóstico de dislocación testicular es tardío y puede pasar desapercibido, documentando la dislocación a su ingreso, varias semanas o años después(11). Es esencial el interrogatorio sobre antecedentes de criptorquidia, retracción testicular o hernioplastía(10), sin embargo, en paciente inconscientes o politraumatizados, esto puede representar una dificultad.

Clínicamente se presenta como un escroto vacío, pero bien desarrollado, el cual es llamado signo de Brockman $(2,8)$, asociado a hematoma, equimosis y edema escrotal o perineal; así mismo se puede palpar una tumoración del tamaño del testículo contralateral, que se puede encontrar dependiendo de la localización del teste dislocado. Es importante el examen físico exhaustivo luego de estabilizar hemodinámicamente al paciente, para su identificación temprana y evitar las posibles complicaciones a corto y largo plazo, como adherencias, hematocele, torsión y necrosis testicular; hipofertilidad secundaria a anticuerpos anti-espermatozoarios, y falta de termorregulación(9-12).

Aunque el diagnóstico es clínico, tradicionalmente se realiza ultrasonido Doppler color, tanto para evaluar la integridad del testículo verificando la continuidad de la túnica albugínea, como para comprobar su flujo vascu- $\operatorname{lar}(6)$, en nuestro caso se realizó ecografía, la cual descartó lesiones a la túnica, así como adecuada vasculatura del cordón espermático, que se confirmó al momento de la exploración testicular. La tomografía computarizada puede utilizarse en casos en los cuales se sospecha que la dislocación es profunda, como en la localización abdominal; así mismo se pueden evaluar otras lesiones a nivel abdominopélvi$\operatorname{co}(2,8,11)$. Su utilización dependerá de su disponibilidad, sin embargo, ningún estudio diagnóstico debería retrasar el tratamiento.

Una vez que la dislocación testicular es diagnosticada, el tratamiento temprano debe ser instituido $(9,11,12)$. El tratamiento de primera línea en varios reportes es la reducción cerrada manual, si la dislocación es diagnosticada de forma temprana, debido a que el edema y las adherencias son mínimas, sin embargo, la tasa de reducción de forma exitosa solamente alcanza el $15 \%$ aproximadamente $(1,2,6,9,11)$; estas maniobras se deben realizar en un área de quirófano bajo sedación o anestesia regional, asistido por ultrasonido Doppler color, para evaluar la indemnidad del flujo vascular y descartar torsión testicular(2). En general el pronóstico es bueno si se realiza de forma precoz, la reposición del testículo(3).

El manejo quirúrgico de la torsión testicular ha ganado mayor interés, principalmente por el bajo porcentaje de éxito en la reducción cerrada, por lo cual, en las revisiones del tema más recientes, se prefiere la exploración testicular como tratamiento inicial(1-3,5,6,10); aunque no es una urgencia estricta, no se debe demorar la intervención quirúrgica, ya que cuanto más tiempo permanezca el testículo en posición ectópica, más daño se producirá y más dificultosa será la cirugía por la fibrosis y presencia de adherencias 1 . La exploración temprana está asociada a incremento de la recuperación y preservación de la fertilidad y la función hormonal(7).

En nuestro caso, se descartaron otras lesiones que ameritaran tratamiento urgente, y se llevó a quirófano para exploración inguinal, en la cual los hallazgos intraoperatorios permitieron 
reducir la dislocación y la orquidopexia transescrotal, presentando adecuada evolución clínica postoperatoria, logrando los objetivos del manejo quirúrgico, que incluyen la exploración, reparación y recuperación testicular(7).

\section{CONCLUSIONES}

La dislocación testicular a pesar de ser una patología poco frecuente, en la actualidad, el aumento del uso de la motocicleta como medio de transporte en nuestro país, predispone a un incremento en las lesiones de los genitales externos que requieren un diagnóstico oportuno y tratamiento quirúrgico temprano para evitar complicaciones a largo plazo.

\section{REFERENCIAS}

1. López-Aramburu, Miguel \& Arias, José \& Pérez, Pablo. (2009). Dislocación (luxación) testicular traumática. Rev Arg de Urologia. Vol. 74.

2. Gómez, Reynaldo G., et al. "Luxación testicular traumática". Urología y Nefrología Internacional, vol. 46, núm. 10, octubre de 2014, págs. $1883-87$.

3. Luján Marco, S., et al. "Dislocación testicular postraumática". Actas Urológicas Españolas, vol. 30, núm. 4, abril de 2006, págs. 409-11. https://doi.org/10.1016/S0210-4806(06)73466-0

4. Bernhard, Zachary, et al. "Testicular Dislocation After Unstable Pelvic Ring Injury”. Cureus, vol. 13, núm. 2. PubMed Central.

5. Granados Loarca, E. A., y V. L. Ambrosio Teni. "Luxación testicular. a propósito de un caso". Actas Urológicas Españolas, vol.24, núm. 4, enero de 2000, pp. 360-61.

https://doi.org/10.1016/S0210-4806(00)72462-4

6. Tai, Yi-Sheng, et al. "Traumatic Testicular Dislocation: A Rare Occurrence of Blunt Scro- tal Injury". Urological Science, vol.25, núm. 4, diciembre de 2014, pp. 158-60.

https://doi.org/10.1016/j.urols.2014.04.001

7. Randhawa, Harkanwal, et al. "Scrotal trauma: A case report and review of the literature". Canadian Urological Association Journal, vol.13, núm. 6S4, 2019. Marzo de 2019. https://doi.org/10.5489/cuaj.5981

8. Riaza Montes, María, et al. "Testicular Dislocation: An Atypical Case and Review of the Literature". Urology Case Reports, vol. 33, noviembre de 2020, p. 101405.

https://doi.org/10.1016/j.eucr.2020.101405

9. Lenfant, Marc, et al. "Traumatic ectopic dislocation of testis: an easily overlooked occurrence of blunt injury in polytrauma patients". Quantitative Imaging in Medicine and Surgery, vol. 9, núm. 12, diciembre de 2019, pp. 200811. https://doi.org/10.21037/qims.2019.11.11

10. Schwartz, Stephen L., y Gary J. Faerber. "Dislocation of the Testis as a Delayed Presentation of Scrotal Trauma".Urology, vol.43, núm.5, mayo de 1994, pp.743-45.

https://doi.org/10.1016/0090-4295(94)90203-8

11. Ko, Sheung-Fat, et al. "Testicular Dislocation". Annals of Emergency Medicine, vol. 43, núm. 3, marzo de 2004, pp. 371-75.

https://doi.org/10.1016/S0196-0644(03)00749-2

12. Feder, Michael, et al. "Testicular Dislocation Following Minor Scrotal Trauma". The American Journal of Emergency Medicine, vol.9, núm.1, enero de 1991, pp. 40-42.

https://doi.org/10.1016/0735-6757(91)90012-9 
Copyright (c) 2021 Andrés Salvador Escobar Gómez, Carlos Gonzalo Estrada Pazos,

Luis Alberto Contreras Morales \& Mario José Jo Bonila

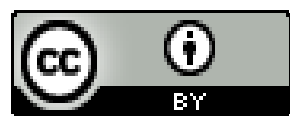

Este texto está protegido por una licencia Creative Commons 4.0.

Usted es libre para Compartir —copiar y redistribuir el material en cualquier medio o formato - y Adaptar el documento — remezclar, transformar y crear a partir del material— para cualquier propósito, , incluso para fines comerciales, siempre que cumpla la condición de:

Atribución: Usted debe dar crédito a la obra original de manera adecuada, proporcionar un enlace a la licencia, e indicar si se han realizado cambios. Puede hacerlo en cualquier forma razonable, pero no de forma tal que sugiera que tiene el apoyo del licenciante o lo recibe por el uso que hace de la obra.

$\underline{\text { Resumen de licencia - Texto completo de la licencia }}$ 